\title{
Low-temperature anharmonic phonon properties of supported graphene
}

\author{
E.A. Kolesov ${ }^{\text {a }}$, M.S. Tivanov ${ }^{\text {a, }}{ }^{*}$, O.V. Korolik $^{\text {a }}$, A.M. Saad ${ }^{\text {b }}$, I.V. Komissarov ${ }^{\text {c }}$ \\ a Belarusian State University, 4 Nezavisimosti Av., 220030 Minsk, Belarus \\ ${ }^{\mathrm{b}}$ Al-Balqa Applied University, PO Box 4545, Amman 11953, Jordan \\ ${ }^{\mathrm{c}}$ Belarusian State University of Informatics and Radioelectronics, 6 P. Brovka, 220013 Minsk, Belarus
}

\section{A R T I C L E I N F O}

Article history:

Received 14 July 2016

Received in revised form

7 October 2016

Accepted 10 October 2016

Available online 18 October 2016

\begin{abstract}
A B S T R A C T
Temperature-dependent Raman studies of anharmonic phonon-phonon processes in supported graphene are presented. Different $G$ peak position temperature dependencies for graphene on glass and copper substrates were observed. Having calculated graphene-substrate total interaction energy taking into account substrate-induced strain, we estimated $E_{2 g}$ mode energy changes due to anharmonic phonon-phonon interaction as the temperature decreases from room temperature to a value close to absolute zero for both substrates. For graphene on glass, the obtained value was about 1.8 times greater than the theoretical one for suspended graphene. For graphene on copper, the value was about 2.9 times greater. This result demonstrates a strong substrate influence on anharmonic phonon-phonon processes in graphene.
\end{abstract}

๑) 2016 Elsevier Ltd. All rights reserved.

\section{Introduction}

Graphene is a promising material for a variety of applications due to its unique physical properties [1]. Among them, one can emphasize an unusually high thermal conductivity [2]. The latter is known to be strongly affected by anharmonic phonon-phonon scattering [3,4], which is important for genuine understanding of thermal transport and other fundamental processes in graphene.

For a number of applications, graphene layers on substrates are needed. Graphene-substrate interfaces, in turn, affect physical properties of graphene [5-10], thus making experimental study of supported graphene layers relevant.

Raman spectroscopy is one of the most powerful tools for graphene studies $[11,12]$. Particularly, analysis of $G$ peak temperature behavior is of great practical importance for determining graphene phonon properties [13].

This paper presents the results of temperature-dependent Raman studies and related analytical calculations performed to establish the substrate influence on $E_{2 g}$ anharmonic properties.

\footnotetext{
* Corresponding author.

E-mail address: tivanov@bsu.by (M.S. Tivanov).
}

\section{Experimental}

Experimental graphene was obtained by atmospheric pressure chemical vapor deposition (CVD).

Prior to the synthesis, copper substrate was electrochemically polished in $1 \mathrm{M}$ phosphoric acid solution for $5 \mathrm{~min}$ with operating voltage of $2.3 \mathrm{~V}$. Synthesis was performed in a tubular quartz reactor with a diameter of $14 \mathrm{~mm}$. During the preliminary treatment, copper foil was annealed at $1050{ }^{\circ} \mathrm{C}$ for $60 \mathrm{~min}$ under the following gas flow rates: hydrogen $-150 \mathrm{cc} / \mathrm{min}$, nitrogen $-100 \mathrm{cc} /$ min. Synthesis was performed under the following conditions: reactor temperature $-1050{ }^{\circ} \mathrm{C}, \mathrm{C}_{10} \mathrm{H}_{22}$ flow rate of $30 \mu \mathrm{L} / \mathrm{min}, \mathrm{N}_{2}$ carrier flow rate of $100 \mathrm{cc} / \mathrm{min}$, synthesis time $-10 \mathrm{~min}$. After the hydrocarbon flow termination, the sample was cooled down to room temperature at a rate of $\sim 50{ }^{\circ} \mathrm{C} / \mathrm{min}$.

Graphene was transferred to glass by wet-chemical room-temperature etching without polymer support in two steps. First, one side (the one that was by reactor wall) of copper foil was treated for 3 min in a solution of $\mathrm{H}_{2} \mathrm{NO}_{3}$ and $\mathrm{H}_{2} \mathrm{O}$ mixed in a volume ratio of $1: 3$. Second, the copper foil was totally dissolved in a water solution of $\mathrm{FeCl}_{3}$. Graphene film was washed several times in a bath with distilled water prior to being placed onto glass.

Raman spectra were obtained with a confocal Raman spectrometer Nanofinder HE (LOTIS TII) with a spectral resolution better than $3 \mathrm{~cm}^{-1}\left(\sim 1.6 \mathrm{~cm}^{-1}\right.$ for $\mathrm{G}$ peak and $\sim 2.7 \mathrm{~cm}^{-1}$ for $2 \mathrm{D}$ peak). For 
Raman radiation excitation, a continuous solid-state laser with a wavelength of $473 \mathrm{~nm}$ was used. Room-temperature Raman measurements were carried out using laser power of $800 \mu \mathrm{W}$, laser spot diameter being of $0.6 \mu \mathrm{m}$.

During low-temperature Raman measurements, the sample was studied in a vacuum (less than $5 \times 10^{-4} \mathrm{~Pa}$ ) temperature-controlled box. Laser power of $5.8 \mathrm{~mW}$ was used, laser spot diameter being of about $1.5 \mu \mathrm{m}$. The measurements were performed in temperature range from 20 to $294 \mathrm{~K}$ with a setting accuracy of $\pm 0.05 \mathrm{~K}$.

AFM measurements were performed using a Solver P47-PRO SPM tool with Z-axis resolution of $0.02 \mathrm{~nm}$.

\section{Results and discussion}

Fig. 1 demonstrates typical room-temperature Raman spectra for graphene on copper and glass substrates. As seen, G and 2D room-temperature Raman peak positions are upshifted for graphene on copper. After transfer to glass substrate, the positions changed by $21 \mathrm{~cm}^{-1}$ and $32 \mathrm{~cm}^{-1}$ for $\mathrm{G}$ and $2 \mathrm{D}$ peaks, respectively. Since the new values were consistent with the literature data for graphene [11,12], we consider doping shift contribution [14-16] to the initially upshifted positions negligible comparing to shift caused by substrate-induced strain $[5,6]$.

In order to determine substrate surfaces roughness, AFM measurements were performed for both copper and glass substrates prepared for graphene synthesis and transfer after all preliminary treatment. As seen from topographic images presented in Fig. 2, both substrate surfaces demonstrate small height variations, copper substrate being slightly more anisotropic for this parameter.

Surface topography parameters statistically obtained from the AFM measurements are presented in Table 1 . Both substrates demonstrate sub-nanometer roughness $R_{a}$, the values of such order being small enough (for example, the roughness range studied in Ref. [17]) and quite close to each other. G peak temperature shift measurements were repeatable for different substrate points, and thus substrate surfaces roughness and anisotropy effects were not considered to strongly affect the $\chi_{\text {str }}$ value.

Fig. 3 presents $G$ peak position temperature dependencies for graphene on both substrates. For copper, a linear dependence with a shift factor of $\chi_{G} \exp =-(5.4 \pm 0.4) \cdot 10^{-2} \mathrm{~cm}^{-1} \mathrm{~K}^{-1}$ was observed. For glass, $G$ peak position remained unchanged throughout the entire

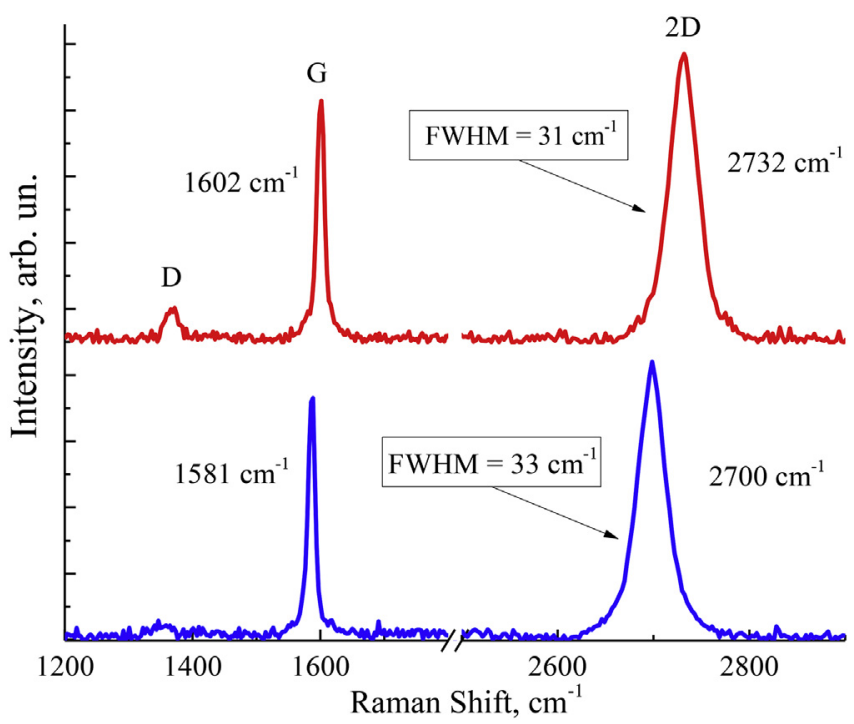

Fig. 1. Typical room-temperature Raman spectra for graphene on copper (top) and glass (bottom) substrates. (A colour version of this figure can be viewed online.) temperature range within the experimental error. Further, we take $\chi_{G \text { exp }} \approx 0 \mathrm{~cm}^{-1} \mathrm{~K}^{-1}$ for graphene on glass.

$G$ peak position temperature shift can be explained by changes in $E_{2 g}$ phonon mode energy due to (i) phonon-phonon contribution $[18,19] \chi_{p h}$; (ii) electron-phonon contribution that changes by less than $1 \mathrm{~cm}^{-1}$ in $0-800 \mathrm{~K}$ temperature range [19] and thus is negligible; (iii) graphene thermal expansion contribution $\chi_{V}$; (iv) graphene deformation caused by thermal expansion coefficients (TECs) difference for graphene and substrate $\chi_{\text {str }}[20,21]$. Thus, phonon-phonon contribution $\chi_{p h}$ can be estimated from the following expression $[20,21]$ :

$\chi_{p h}=\chi_{G \exp }-\chi_{V}-\chi_{s t r}$.

It is important to note that similar analysis for wider temperature range (i.e. including high-temperature measurements) should also take into account a dramatic change from hole to electron electrostatic doping of graphene [22].

Thermal expansion contribution to the observed $G$ peak temperature shift can be described as [20]:

$$
\begin{aligned}
\chi_{V}= & \frac{\omega(0)}{T_{1}-T_{2}}\left[\exp \left(-3 \gamma_{E_{2 g}} \int_{0}^{T_{1}} \alpha_{G}\left(T^{\prime}\right) d T^{\prime}\right)\right. \\
& \left.-\exp \left(-3 \gamma_{E_{2 g}} \int_{0}^{T_{2}} \alpha_{G}\left(T^{\prime}\right) d T^{\prime}\right)\right]
\end{aligned}
$$

where $\gamma_{E_{2 g}}$ is a Grüneisen parameter for an optical phonon from the center of Brillouin zone (in this paper value of $\gamma_{E_{2 g}}=1.8$ [23] was used), $\alpha_{G}\left(T^{\prime}\right)$ is a thermal expansion coefficient for graphene as a function of temperature $T^{\prime}$.

The influence of mechanical stress caused by difference in thermal expansion coefficients for graphene and substrate can be written as [24]:

$$
\chi_{s t r}=\frac{\beta}{\Delta T} \int_{294 K}^{T}\left(\alpha_{s u b}\left(T^{\prime}\right)-\alpha_{G}\left(T^{\prime}\right)\right) \mathrm{d} T^{\prime}
$$

where $\beta=\partial \omega_{G} / \partial \varepsilon$ is a biaxial strain rate, $\varepsilon$ is a relative deformation (\%), $\alpha_{\text {sub }}\left(T^{\prime}\right)$ and $\alpha_{G}\left(T^{\prime}\right)$ are the thermal expansion coefficients as functions of temperature $T^{\prime}$ for substrate and graphene, respectively.

At a room temperature, graphene thermal expansion coefficient is $\alpha_{G} \sim-8 \cdot 10^{-6} \mathrm{~K}$ [24]. Due to its negative value, atypical for most materials, the amount of mechanical strain caused by TECs difference for graphene and the substrate can't be negligible for a wide variety of conventional substrates. Besides, $\alpha_{G}$ is known to depend on temperature, with this dependence being strongly affected by the substrate, and therefore simply considering TECs difference in order to obtain the amount of mechanical strain is not enough. In particular, the case of a sufficiently strong interaction of graphene with the substrate is possible, wherein $\alpha_{G}$ value can be positive in the whole temperature range [8], leading to contraction of graphene lattice rather than expansion, as the temperature decreases. Thus, the correct calculation of $\chi_{p h}$ using (1-3) requires close examination of graphene-substrate interaction in order to estimate its energy and to choose the correct $\alpha_{G}\left(T^{\prime}\right)$ dependencies [8].

In order to compare copper and glass interaction with graphene, an approach by Aitken and Huang [25] was used. The estimation was carried out in the approximation of homogeneous substrate and defect-free graphene-substrate interface.

Van-der-Waals interaction potential between graphene and 

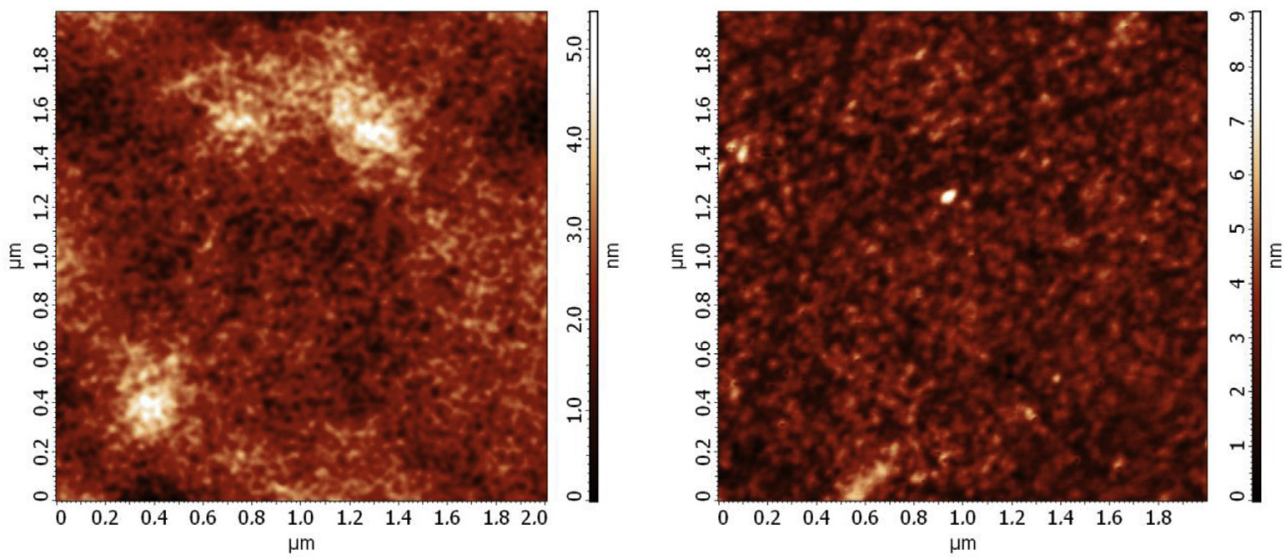

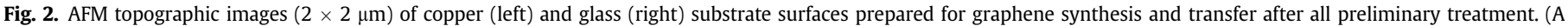
colour version of this figure can be viewed online.)

Table 1

Average values obtained from AFM measurements and defining morphology of the substrate surfaces.

\begin{tabular}{lll}
\hline Substrate & Copper & Glass \\
\hline Arithmetic mean surface height $\bar{z}, \mathrm{~nm}$ & 2.21 & 2.20 \\
Arithmetic mean deviation of the roughness profile $R_{a}, \mathrm{~nm}$ & 0.46 & 0.51 \\
Root mean square deviation of the roughness profile $R_{q}, \mathrm{~nm}$ & 0.62 & 0.68 \\
\hline
\end{tabular}

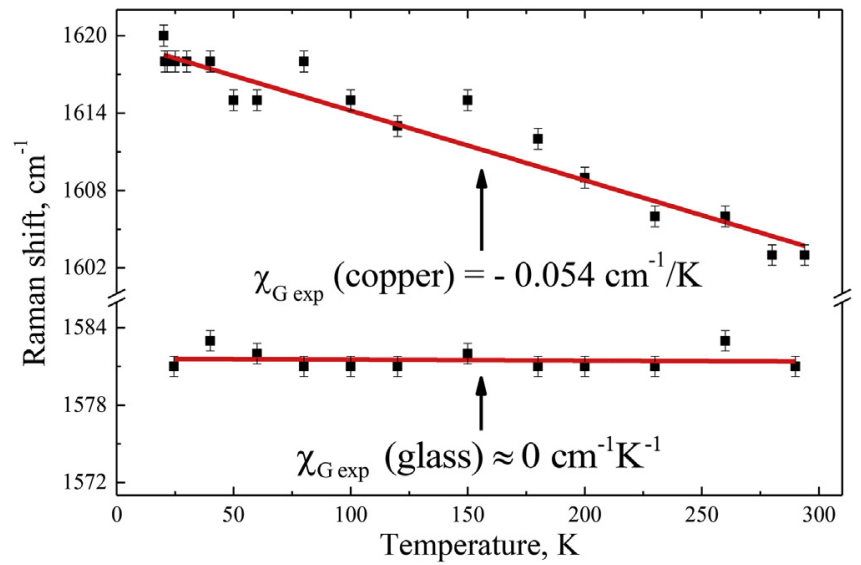

Fig. 3. G peak position temperature dependencies for graphene on copper (top) and glass (bottom) substrates. Squares represent experimental data with measurement error indicated, lines show the linear approximation. (A colour version of this figure can be viewed online.)

substrate can be written as a function of graphene-substrate distance $z[25,26]$ :

$U_{v d W}(z)=-\Gamma_{0}\left(\frac{3 h_{0}^{3}}{2 z^{3}}-\frac{h_{0}^{9}}{2 z^{9}}\right)$,

where $h_{0}$ is the equilibrium separation distance and $\Gamma_{0}$ is the adhesion energy per unit area. Here we take the values of $\Gamma_{0}$ for copper and glass $0.72 \mathrm{~J} / \mathrm{m}^{2}$ [27] and $0.45 \mathrm{~J} / \mathrm{m}^{2}$ [28], respectively.

The authors of [22] reported distance fluctuations present in the graphene-substrate system. The amplitude decreased for lower temperatures, and thus is expected to be minimal for values close to absolute zero. The fluctuations should be taken into account when considering average graphene-substrate distance during a similar calculation for high temperatures.

Here we assume the average equilibrium separations $h_{0}$ of $0.300 \mathrm{~nm}$ for glass substrate [29] and of $0.326 \mathrm{~nm}$ for copper substrate [30]. The minimum value of $U_{v d W}$ corresponds to $z=h_{0}$, and $\Gamma_{0}$ corresponds to the energy well depth at $h_{0}$. Parameters $\Gamma_{0}$ and $h_{0}$ are related to Lennard-Jones constants as

$$
\begin{aligned}
& h_{0}=\left(\frac{2 C_{2}}{5 C_{1}}\right)^{\frac{1}{6}}, \\
& \Gamma_{0}=\frac{\pi C_{1} \rho_{s} \rho_{g}}{9 h_{0}^{3}},
\end{aligned}
$$

where $C_{1}$ and $C_{2}$ are the repulsive and attractive interaction constants, $\rho_{s}$ and $\rho_{g}$ are the numbers of atoms per unit volume of the substrate and per unit area of graphene, respectively.

In the context of graphene-substrate interaction analysis, substrate-induced strain can be taken into account in terms of graphene corrugations caused by mechanical stresses induced. Assuming a linear sinusoidal corrugation $z(x)=h_{0}+A_{G} \sin (2 \pi x / \lambda)$, the total energy per unit area of graphene includes two terms. The first term is van der Waals energy of corrugated graphene, obtained integrating graphene-substrate interaction potential over a corrugation wavelength. The second term is the elastic strain energy. Thus, the total energy can be expressed as [25]:

$$
\begin{aligned}
E_{\text {total }}= & \Gamma_{0}\left(-1+\frac{A_{G}^{2}}{4 h_{0}^{2}}\left[27+\frac{C \varepsilon}{\Gamma_{0}}\left(\frac{2 \pi h_{0}}{\lambda}\right)^{2}+\frac{D}{\Gamma_{0} h_{0}^{2}}\left(\frac{2 \pi h_{0}}{\lambda}\right)^{4}\right]\right. \\
& \left.+\frac{A_{G}^{4}}{8 h_{0}^{4}}\left[675+\frac{3 C}{8 \Gamma_{0}}\left(\frac{2 \pi h_{0}}{\lambda}\right)^{4}\right]\right),
\end{aligned}
$$

where $C$ is the two-dimensional in-plane elastic modulus, $D$ is the bending modulus for graphene. By minimizing the total energy (setting $\frac{\partial E_{\text {total }}}{\partial A_{C}}=0$ and $\frac{\partial E_{\text {total }}}{\partial \lambda}=0$ ), equilibrium corrugation wavelength $\lambda$ and amplitude $A_{G}$ can be determined for a known amount of strain $\varepsilon$, leading to determination of $E_{\text {total }}$.

Room-temperature biaxial strain in graphene on copper, having emerged due to lattice mismatch or during the cooling stage of sample preparation (the roughness was considered negligible based on AFM results), can be obtained from G peak roomtemperature shift $\Delta \omega_{G}$ with relation to the normal position using the following expression [31]: 
$\varepsilon=-\frac{\Delta \omega_{G}}{2 \omega_{G}^{0} \gamma_{E_{2 g}}}$,

where $\omega_{G}^{0}=1581 \mathrm{~cm}^{-1}$ is the normal G peak room-temperature position. Due to normal positions of room-temperature Raman peaks for glass substrate, a value of $\varepsilon=0$ was used in calculation of corresponding $E_{\text {total }}(294 \mathrm{~K})$.

Since several processes influence $G$ peak position temperature shift, low-temperature biaxial strain was obtained by integration of thermal expansion coefficients difference as in formula (3). For copper, thermal expansion temperature dependence $\alpha_{\mathrm{Cu}}\left(T^{\prime}\right)$ from Ref. [32] was used. Since thermal expansion coefficient of glass we used was very close to that of amorphous silicon, $\alpha_{\mathrm{Si}}\left(T^{\prime}\right)$ dependence from Ref. [33] was chosen for our estimation. Since we did not know yet which $\alpha_{G}\left(T^{\prime}\right)$ dependence from Ref. [8] should be used for our case, we performed $E_{\text {total }}$ calculation for all dependencies presented there.

Calculated total interaction energy for copper was $0.83 \mathrm{~J} / \mathrm{m}^{2}$, and for glass $E_{\text {total }}$ value turned out to be $0.51 \mathrm{~J} / \mathrm{m}^{2}$. According to calculation results, the total interaction energy turned out to change only in the third digit after the decimal point as the temperature decreased from $294 \mathrm{~K}$ to a value close to absolute zero for graphene on both substrates: $E_{\text {total }}(294 \mathrm{~K})-E_{\text {total }}(0 \mathrm{~K}) \approx 0.003 \mathrm{~J} / \mathrm{m}^{2}$. This change is out if the calculation accuracy. Moreover, $E_{\text {total }}$ value does not substantially change in case of using any dependence from Ref. [8], indicating that strain increase due to thermal expansion coefficients mismatch does not strongly affect graphene-substrate interaction energy within the utilized approach (but that does not mean that the impact on $\chi_{p h}$ is also negligible!). Thus, $E_{\text {total }}$ defines $\alpha_{G}\left(T^{\prime}\right)$, but $\alpha_{G}\left(T^{\prime}\right)$ does not have a strong impact on $E_{\text {total }}$ in terms of taking into account the substrate-induced strain.

Calculated $E_{\text {total }}$ values allow to choose the correct temperature dependencies for graphene thermal expansion coefficient. Assuming that $E_{\text {total }}$ difference for both substrates is considered in $\alpha_{G}\left(T^{\prime}\right)$ dependencies and therefore in equations (2) and (3), $\chi_{p h}$ can be finally estimated from equations (1)-(3) using $\alpha_{G}\left(T^{\prime}\right)$ dependencies for our interaction energies from Ref. [8]. It turned out that for both substrates graphene behavior is mostly substratedominated at higher temperatures, and in that temperature range graphene lattice contracts as the temperature decreases: the $\alpha_{G}\left(T^{\prime}\right)$ parameter becomes zero at about $190 \mathrm{~K}$ and $225 \mathrm{~K}$ for glass and copper substrates, respectively. As the cooling continues, graphene TEC becomes negative, leading to the lattice-dominated expansion of the material.

Stronger graphene interaction with crystalline material - copper - leads to more narrow temperature range in which $\alpha_{G}\left(T^{\prime}\right)$ is negative, comparing to amorphous glass. This leads to lower absolute values of integrals in expressions (2) and (3), signifying that graphene contracts less before it starts to expand as the temperature rises. For glass, $\chi_{V}$ and $\chi_{s t r}$ terms have greater values enough to nullify $\chi_{p h}$, resulting in the absence of experimentally measured shift.

For graphene on glass substrate the obtained phonon-phonon scattering term is $\chi_{p h}=-\left(4.9_{ \pm} 0.6\right) \cdot 10^{-2} \mathrm{~cm}^{-1} \mathrm{~K}^{-1}$. This value corresponds to $E_{2 g}$ phonon mode energy changes (due to anharmonic phonon-phonon scattering contribution) value of $\Delta E \approx 1.77 \cdot 10^{-3} \mathrm{eV}\left(14.3 \mathrm{~cm}^{-1}\right.$ shift $)$ as the temperature decreases from $294 \mathrm{~K}$ to a value close to absolute zero, this value being approximately 1.8 times greater than that computed in Ref. [19] without taking into account substrate influence.

For the case of copper substrate our estimations give $\chi_{p h}=-(7.6 \pm 0.7) \cdot 10^{-2} \mathrm{~cm}^{-1} \mathrm{~K}^{-1}$. It corresponds to $\Delta E \approx 2.78 \cdot 10^{-3} \mathrm{eV}\left(22.4 \mathrm{~cm}^{-1}\right.$ shift $)$ as the temperature decreases from $294 \mathrm{~K}$ to absolute zero limit. Obtained $\Delta E$ is about 2.9 times greater than in Ref. [19].

The obtained difference of phonon-phonon scattering terms and their discrepancy with calculations for free-standing graphene demonstrate strong substrate influence on $\mathrm{E}_{2 \mathrm{~g}}$ phonon processes in graphene within the utilized approach. The substrate possibly affects the phase space of anharmonic phonon-phonon scattering, changing its contribution to $G$ peak temperature shift and its effect on graphene thermal conductivity, which is dominated by phononphonon interactions [34]. This result also demonstrates the importance of further investigation of supported graphene anharmonic phonon properties.

\section{Conclusion}

During low-temperature Raman measurements for graphene on copper, a linear $G$ peak position temperature dependence was observed. For graphene on glass, G peak position remained unchanged throughout the whole temperature range of $20-294 \mathrm{~K}$ within the experimental error. In order to understand the difference in graphene interactions with glass and copper, maximum van der Waals interaction force and total interaction energy were calculated taking into account graphene corrugations caused by substrate-induced strain for both substrates.

Using the obtained values, $E_{2 g}$ mode energy changes due to anharmonic phonon-phonon interaction as the temperature decreases from 294 to a value close to absolute zero were estimated for both substrates. The obtained value was about 1.8 times greater than the theoretical one for graphene on glass and about 2.9 times greater for graphene on copper. This result demonstrates strong substrate influence on anharmonic phonon-phonon processes in graphene.

\section{References}

[1] Y. Zhu, S. Murali, W. Cai, X. Li, J.W. Suk, J.R. Potts, et al., Graphene and graphene oxide: synthesis, properties, and applications, Adv. Mater. 22 (2010) 3906-3924, http://dx.doi.org/10.1002/adma.201001068.

[2] A.A. Balandin, S. Ghosh, W. Bao, I. Calizo, D. Teweldebrhan, F. Miao, et al., Superior thermal conductivity of single-layer graphene, Nano Lett. 8 (2008) 902-907, http://dx.doi.org/10.1021/nl0731872.

[3] L. Lindsay, D.A. Broido, N. Mingo, Flexural phonons and thermal transport in graphene, Phys. Rev. B 82 (2010) 115427, http://dx.doi.org/10.1103/ PhysRevB.82.115427.

[4] L. Lindsay, Wu Li, J. Carrete, N. Mingo, D.A. Broido, T.L. Reinecke, Phonon thermal transport in strained and unstrained graphene from first principles, Phys. Rev. B 89 (2014) 155426, http://dx.doi.org/10.1103/ PhysRevB.89.155426.

[5] O. Frank, J. Vejpravova, V. Holy, L. Kavan, M. Kalbac, Interaction between graphene and copper substrate: the role of lattice orientation, Carbon 68 (2014) 440-451, http://dx.doi.org/10.1016/j.carbon.2013.11.020.

[6] R. He, L. Zhao, N. Petrone, K.S. Kim, M. Roth, J. Hone, et al., Large physisorption strain in chemical vapor deposition of graphene on copper substrates, Nano Lett. 12 (2012) 2408-2413, http://dx.doi.org/10.1021/nl300397v.

[7] S. Yu Davydov, Estimating anharmonic characteristics of single-sheet graphene at high temperatures, Tech. Phys. Lett. 37 (2011) 1161-1164, http:// dx.doi.org/10.1134/S1063785011120194.

[8] J. Jiang, J. Wang, B. Li, Thermal expansion in single-walled carbon nanotubes and graphene: nonequilibrium Green's function approach, Phys. Rev. B 80 (2009) 205429, http://dx.doi.org/10.1103/PhysRevB.80.205429.

[9] Y. Wang, Z. Ni, T. Yu, Z.X. Shen, H. Wang, Y. Wu, et al., Raman studies of monolayer graphene: the substrate effect, J. Phys. Chem. C 112 (2008) 10637-10640, http://dx.doi.org/10.1021/jp8008404.

[10] I. Calizo, S. Ghosh, W. Bao, F. Miao, C.N. Lau, A.A. Balandin, Raman nanometrology of graphene: temperature and substrate effects, Solid St. Commun. 149 (2009) 1132-1135, http://dx.doi.org/10.1016/j.ssc.2009.01.036.

[11] A.C. Ferrari, J.C. Meyer, V. Scardaci, C. Casiraghi, M. Lazzeri, F. Mauri, et al. Raman spectrum of graphene and graphene layers, Phys. Rev. Lett. 97 (2006) 187401, http://dx.doi.org/10.1103/PhysRevLett.97.187401.

[12] A.C. Ferrari, D.M. Basko, Raman spectroscopy as a versatile tool for studying the properties of graphene, Nat. Nanotechnol. 8 (2013) 235-246, http:// dx.doi.org/10.1038/nnano.2013.46.

[13] I. Calizo, A.A. Balandin, W. Bao, F. Miao, C.N. Lau, Temperature dependence of the Raman spectra of graphene and graphene multilayers, Nano Lett. 7 (2007) 2645-2649, http://dx.doi.org/10.1021/nl071033g. 
[14] M. Kalbac, A. Reina-Cecco, H. Farhat, J. Kong, L. Kavan, M.S. Dresselhaus, The influence of strong electron and hole doping on the Raman intensity of chemical vapor-deposition graphene, ACS Nano 4 (2010) 6055-6063, http:/ dx.doi.org/10.1021/nn1010914.

[15] S. Pisana, M. Lazzeri, C. Casiraghi, K.S. Novoselov, A.K. Geim, A.C. Ferrari, et al., Breakdown of the adiabatic Born-Oppenheimer approximation in graphene, Nat. Mater. 6 (2007) 198-201, http://dx.doi.org/10.1038/nmat1846.

[16] A. Das, S. Pisana, B. Chakraborty, S. Piscanec, S.K. Saha, U.V. Waghmare, et al., Monitoring dopants by Raman scattering in an electrochemically top-gated graphene transistor, Nat. Nanotechnol. 3 (2008) 210-215, http://dx.doi.org 10.1038/nnano.2008.67.

[17] H. Pourzand, M. Tabib-Azar, Graphene thickness dependent adhesion force and its correlation to surface roughness, Appl. Phys. Lett. 104 (2014) 171603 , http://dx.doi.org/10.1063/1.4871315.

[18] M. Lazzeri, M. Calandra, F. Mauri, Phys. Rev. B 68 (2003) 220509(R).

[19] N. Bonini, M. Lazzeri, N. Marzari, F. Mauri, Phonon anharmonicities in graphite and graphene, Phys. Rev. Lett. 99 (2007) 176802, http://dx.doi.org/10.1103/ PhysRevLett.99.176802.

[20] C. Postmus, J.R. Ferraro, Pressure dependence of infrared eigenfrequencies of $\mathrm{KCl}$ and $\mathrm{KBr}$, Phys. Rev. 174 (1968) 983-987, http://dx.doi.org/10.1103/ PhysRev.174.983.

[21] J. Lin, L. Guo, Q. Huang, Y. Jia, K. Li, X. Lai, et al., Anharmonic phonon effects in Raman spectra of unsupported vertical graphene sheets, Phys. Rev. B 83 (2011) 125430, http://dx.doi.org/10.1103/PhysRevB.83.125430w.

[22] S. Ulstrup, M. Bianchi, R. Hatch, D. Guan, A. Baraldi, D. Alfè, et al., High-temperature behavior of supported graphene: electron-phonon coupling and substrate-induced doping, Phys. Rev. B 93 (2016) 239901, http://dx.doi.org 10.1103/PhysRevB.86.161402.

[23] T.M.G. Mohiuddin, A. Lombardo, R.R. Nair, A. Bonetti, G. Savini, R. Jalil, et al., Uniaxial strain in graphene by Raman spectroscopy: G peak splitting, Grüneisen parameters, and sample orientation, Phys. Rev. B 79 (2009) 205433, http://dx.doi.org/10.1103/PhysRevB.79.205433.

[24] D. Yoon, Y. Son, H. Cheong, Negative thermal expansion coefficient of graphene measured by Raman spectroscopy, Nano Lett. 11 (2011) 3227-3231, http://dx.doi.org/10.1021/nl201488g.

[25] Z.H. Aitken, R. Huang, Effects of mismatch strain and substrate surface corrugation on morphology of supported monolayer graphene, J. Appl. Phys. 107 (2010) 123531, http://dx.doi.org/10.1063/1.3437642.

[26] J.N. Israelachvili, Intermolecular and Surface Forces, second ed., Academic Press, Lincoln, 1992.

[27] T. Yoon, W.C. Shin, T.Y. Kim, J.H. Mun, T.-S. Kim, B.J. Cho, Direct measurement of adhesion energy of monolayer graphene as-grown on copper and its application to renewable transfer process, Nano Lett. 12 (2012) 1448-1452, http://dx.doi.org/10.1021/nl204123h.

[28] S.P. Koenig, N.G. Boddeti, M.L. Dunn, J.S. Bunch, Ultrastrong adhesion of graphene membranes, Nat. Nanotechnol. 6 (2011) 543-546, http://dx.doi.org/ 10.1038/nnano.2011.123.

[29] M.Z. Hossain, Chemistry at the graphene-SiO2SiO2 interface, Appl. Phys. Lett. 95 (2009) 143125, http://dx.doi.org/10.1063/1.3247964.

[30] Z. Xu, M.J. Buehler, Interface structure and mechanics between graphene and metal substrates: a first-principles study, J. Phys. Condens. Matter 22 (2010) 485301, http://dx.doi.org/10.1088/0953-8984/22/48/485301.

[31] N. Ferralis, Probing mechanical properties of graphene with Raman spectroscopy, J. Mater. Sci. 45 (2010) 5135-5149, http://dx.doi.org/10.1007/ s10853-010-4673-3.

[32] M. Okaji, N. Yamada, H. Kato, K. Nara, Measurements of linear thermal expansion coefficients of copper SRM 736 and some commercially available coppers in the temperature range $20-300 \mathrm{~K}$ by means of an absolute interferometric dilatometer, Cryogenics 37 (1997) 251-254, http://dx.doi.org/ 10.1016/S0011-2275(97)00010-6.

[33] J. Fabian, P.B. Allen, Thermal expansion and Grüneisen parameters of amorphous silicon: a realistic model calculation, Phys. Rev. Lett. 79 (1997) 1885, http://dx.doi.org/10.1103/PhysRevLett.79.1885.

[34] J.H. Warner, F. Schäffel, A. Bachmatiuk, M.H. Rümmeli, Graphene: Fundamentals and Emergent Applications, Elsevier, New York, 2012. 